\title{
Association between hyperlipidemia and chronic kidney disease in a Japanese population; a cross- sectional study
}

Yuko AGO SHIRAISHI ( $\square$ agoyuko818@jichi.ac.jp )

Jichi Medical University School of Medicine Graduate School of Medicine: Jichi Ika Daigaku Igakubu Daigakuin Igaku Kenkyuka https://orcid.org/0000-0002-6087-0855

\section{Yukiko ISHIKAWA}

Jichi Medical University: Jichi lka Daigaku

Joji ISHIKAWA

Tokyo Metropolitan Geriatric Hospital and Institute of Gerontology: Tokyo-to Kenko Choju Iryo Center

\section{Masami MATSUMURA}

Jichi Medical University: Jichi lka Daigaku

Shizukiyo ISHIKAWA

Jichi Medical University: Jichi Ika Daigaku

\section{Research article}

Keywords: chronic kidney disease, prevalence, hyperlipidemia, hypertriglyceridemia

Posted Date: March 19th, 2021

DOI: https://doi.org/10.21203/rs.3.rs-151545/v2

License: (c) (1) This work is licensed under a Creative Commons Attribution 4.0 International License.

Read Full License 


\section{Abstract}

Background: Strategies to prevent the development and progression of chronic kidney disease (CKD) are important in clinical practice due to increased life expectancy. The present study investigated the prevalence of CKD as well as lipid profiles affecting CKD, especially triglyceride (TG) levels.

Methods: In total, 5,169 subjects were eligible for a cross-sectional analysis of baseline data from the Jichi Medical School Cohort Study. We examined CKD subjects with an estimated glomerular filtration rate (eGFR) of $59 \mathrm{~mL} / \mathrm{min} / 1.73 \mathrm{~m}^{2}$ or lower and independent factors associated with reductions in eGFR.

Results: The prevalence of CKD was $17.7 \%$. Age, systolic blood pressure, and hyperlipidemia were defined as related factors for CKD. The lowest, second, third, and highest quartile ranges of total cholesterol (TC) and TG were 0-166, 167-188, 189-212, and $213 \mathrm{mg} / \mathrm{dL}$ or higher and 0-71, 72-100, 101-148, and 149 $\mathrm{mg} / \mathrm{dL}$ or higher, respectively. The odds ratio (OR) of Q2 to Q4 of TC relative to that of Q1 for CKD increased linearly (OR [95\%Cl]: Q2, 1.3 [1.0-1.7]; Q3, 1.38 [1.1-1.8]; Q4, 1.5 [1.4-2.4]). The ORs of Q2 and Q3 of TG for CKD did not increase, whereas that of Q4 did (OR [95\% Cl]: Q2, 0.95 [0.7-1.2]; Q3, 0.98 [0.8-1.2]; Q4, 1.21 [1.0-1.5]).

Conclusion: Increases in TC and TG levels were both independently associated with CKD. The relationship with CKD became stronger as TC increased, and the TG had threshold was $149 \mathrm{mg} / \mathrm{dL}$.

\section{Background}

Chronic kidney disease (CKD) is a global problem in clinical practice due to increased life expectancy. In $2005,13 \%$ of Japanese adults who participated in the annual health check program were reported to have CKD. [1] Another cohort study in Japan showed that the prevalence of CKD was increasing. [2] The progression of CKD ultimately results in end-stage renal disease and dialysis. In Japan, 339,841 patients were receiving dialysis in 2018 and this number has been increasing by approximately 5,000 patients annually. [3]

Many studies investigated the association between hyperlipidemia and CKD and the parameters of hyperlipidemia. Although metabolic syndrome (MetS), and total cholesterol (TC) are well-known risks, the association with triglycerides (TG) currently remains unclear. [4] MetS consists of at least three of the following five disorders: abdominal obesity, hypertriglyceridemia, low high-density lipoprotein cholesterol (HDL-C), hypertension, and hyperglycemia. A systematic review identified MetS as a predictor of the development of CKD. [5] Schaeffner et al. demonstrated in a prospective study that renal dysfunction was more common in men with TC greater than $240 \mathrm{mg} / \mathrm{dL}$. [6] However, the threshold level of TG that affects the development of CKD has not yet been established. Limited information is currently available on the relationship between TG levels and CKD. In a Chinese cross-sectional study, increases in TG levels were linearly associated with mild declines in renal function in subjects with an estimated glomerular filtration rate (eGFR) of between 60 and $90 \mathrm{~mL} / \mathrm{min} / 1.73 \mathrm{~m}^{2}$. [7] Another cross-sectional study in Taiwan reported 
that TG levels higher than $200 \mathrm{mg} / \mathrm{dL}$ were associated with the development of CKD in subjects recruited from a medical screening program. [8]

Herein, we investigated lipid profiles related to CKD, especially the TG level related to CKD, in a crosssectional study of Japanese community-based residents. The aim of this study was to investigate the frequency of CKD and the association of CKD-related factors, especially lipids and CKD. We conducted a cross-sectional analysis of baseline data from the Jichi Medical School (JMS) Cohort Study.

\section{Methods}

\section{Subjects}

The objective of the JMS Cohort Study was to evaluate the relationship between risk factors and cardiovascular diseases (CVD) in a general Japanese population. Details on the JMS cohort have been described previously. [9] Subjects were recruited from mass screening examinations for CVD by the Health and Medical Service Law for the Aged conducted by 11 communities between April 1992 and July 1995. The baseline data of this cohort were used in this cross-sectional study. The total number of subjects in the cohort was 12,490 (4,913 men and 7,577 women) and they were aged between 19 and 93 years. After the exclusion of subjects whose serum creatinine ( $\mathrm{SCr}$ ) value was not obtained, 5,169 subjects (1,870 men and 3,299 women) remained eligible for the CKD study.

\section{Variables}

Baseline information on age, sex, body mass index (BMI), systolic blood pressure (SBP), diastolic blood pressure (DBP), blood sugar (BS), TC, and TG was collected. Information on the smoking and drinking status as well as previous medical histories were obtained from questionnaires. Diabetes was defined as fasting $B S$ (FBS) $\geq 126 \mathrm{mg} / \mathrm{dL}$ and/or casual BS $\geq 200 \mathrm{mg} / \mathrm{dL}$ and receiving medication for diabetes. Hyperlipidemia was defined as TC $\geq 220 \mathrm{mg} / \mathrm{dL}$ and/or $\mathrm{TG} \geq 150 \mathrm{mg} / \mathrm{dL}$ and receiving medication for hyperlipidemia. We calculated eGFR according to the modification of diet in renal disease eGFR (MDRDeGFR) for Japanese using the following equations: eGFR in men $=194 \times \mathrm{SCr}^{-1.094} \times \mathrm{Age}^{-0.287}$, while eGFR in women $=194 \times \mathrm{SCr}^{-1.094} \times \mathrm{Age}^{-0.287} \times 0.739$. [10] Subjects were classified into CKD stages based on the definition of the Kidney Disease Outcomes Quality Initiative according to eGFR. [11] In the present study, subjects with eGFR less than $60\left(\mathrm{~mL} / \mathrm{min} / 1.73 \mathrm{~m}^{2}\right)$ were defined as the CKD group.

\section{Statistical analysis}

Continuous variables were expressed as means and standard deviations (SD). Categorical variables were expressed as percentages (\%). The differences in mean values between CKD and non-CKD were calculated using the $t$-test, while differences in percentages were calculated using the $\chi^{2}$ test. We performed a multiple linear regression analysis to assess the relationships between various factors and reductions in eGFR adjusted for age, sex, BMI, SBP, hyperlipidemia, diabetes, and current smoking and alcohol habits. $\beta$, standard errors (SE), and $P$ values were calculated using this model. We performed a 
logistic regression analysis to evaluate related factors for the CKD group. In addition, we calculated odds ratios (OR), $95 \%$ confidence intervals $(95 \% \mathrm{Cl})$, and $\mathrm{P}$ values adjusted for age, sex, $\mathrm{BMI}, \mathrm{SBP}$, the category of hyperlipidemia, diabetes, and current smoking and alcohol habits. To assess lipid profile risks, we used $T G \geq 150 \mathrm{mg} / \mathrm{dL}, T C \geq 220 \mathrm{mg} / \mathrm{dL}$, and both $\mathrm{TC} \geq 220 \mathrm{mg} / \mathrm{dL}$ and $\mathrm{TG} \geq 150 \mathrm{mg} / \mathrm{dL}$ as independent variables as categories of hyperlipidemia. We then examined the $\mathrm{OR}$ of the quartiles of TC and TG for CKD adjusted for age, sex, BMI, SBP, diabetes, and current smoking and alcohol habits by a logistic regression analysis.

Statistical analyses were performed using SPSS ver. 21 (IBM SPSS Statistics 21.0).

\section{Results}

The total number of subjects was 5,169 and $36.2 \%$ were men. The mean age of subjects was 53.9 ([SD] 11.2) years, 53.6 (11.4) years for men, 54.1 (11.0) years for women. The prevalence of CKD was $17.7 \%$ ( $15.7 \%$ in men, $18.9 \%$ in women). The numbers of subjects with eGFR of $90 \mathrm{~mL} / \mathrm{min} / 1.73 \mathrm{~m}^{2}$ or higher, $60-89 \mathrm{~mL} / \mathrm{min} / 1.73 \mathrm{~m}^{2}, 30-59 \mathrm{~mL} / \mathrm{min} / 1.73 \mathrm{~m}^{2}$, or $\leq 30 \mathrm{~mL} / \mathrm{min} / 1.73 \mathrm{~m}^{2}$ were $1,194(23.1 \%), 3,058$ (59.2\%), 913 (17.7\%), and $4(0.08 \%)$ respectively. The number of subjects with positive proteinuria was 67 $(1.3 \%)$. Because the percentage of those subjects was very small, we did not use the findings of proteinuria for the definition of CKD. The number of subjects with hyperlipidemia was $1,891(36.6 \%)$, of which $85(1.7 \%)$ had been treated. The number of the subjects with the TG $\geq 150 \mathrm{mg} / \mathrm{dL}$ alone, TC $\geq 220$ $\mathrm{mg} / \mathrm{dL}$ alone, or both $\mathrm{TC} \geq 220 \mathrm{mg} / \mathrm{dL}$ and TG $\geq 150 \mathrm{mg} / \mathrm{dL}$ were 860 (16.7\%), 610 (11.8\%), and 410 $(0.79 \%)$, respectively. The prevalence of CKD in each age group of 30-39, 40-49, 50-59, and $\geq 60$ years were $0.8,7.6,11.5$, and $27.3 \%$ in men and $0.4,5.8,23.1$, and $27.9 \%$ in women, respectively (Figure 1 ).

Table 1 shows the general characteristics of CKD and non-CKD subjects. Age, the percentage of men, BMI, SBP, DBP, TC, TG, hyperlipidemia, and BS were higher in the CKD group than in the non-CKD group. No significant differences were observed in the prevalence of diabetes between the two groups. The percentage of subjects with current smoking and alcohol habits was lower in the CKD group than in the non-CKD group.

Table 2 shows that age $(p<0.001)$, sex $(p<0.05)$, SBP $(p<0.001)$, and hyperlipidemia $(p<0.001)$ were associated with reductions in eGFR in a multiple linear regression analysis model.

Table 3 shows the OR for the CKD group adjusted for multiple variables. Age (OR [95\%Cl]: 1.07, [1.061.09]), SBP (1.01, [1.00-1.01]), TG $\geq 150 \mathrm{mg} / \mathrm{dL}$ alone (1.34, [1.07-1.66]), TC $\geq 220 \mathrm{mg} / \mathrm{dL}$ alone (1.55, [1.23-1.94]), and high TG and TC (1.94, [1.48-2.54]) correlated with CKD. An additive effect of TG and TC on CKD was observed (data not shown). The synergistic effect of TG and TC on CKD was also noted in this regression model in another evaluation.

Figure 2 shows the $\mathrm{OR}$ of TC and TG in quartiles for CKD adjusted according to multiple variables. The lowest, second, third, and highest quartile ranges of total TC and TG were $0-166,167-188,189-212$, and $213 \mathrm{mg} / \mathrm{dL}$ or higher and 0-71, 72-100, 101-148, and $149 \mathrm{mg} / \mathrm{dL}$ or higher, respectively. The OR of Q2 to 
Q4 of TC relative to Q1 for CKD increased linearly (OR [95\%Cl]: Q2, 1.3 [1.0-1.7]; Q3, 1.38 [1.1-1.8]; Q4, 1.5 [1.4-2.4]). Although the OR of Q2 and Q3 of TG for CKD did not increase, the OR of Q4 of TG for CKD was significantly higher than that for Q1 (OR [95\% Cl]: Q2, 0.95 [0.7-1.2]; Q3, 0.98 [0.8-1.2]; Q4, 1.21 [1.0-1.5]). The OR according to TG elevations significantly increased only in the highest quartile valued at 149 $\mathrm{mg} / \mathrm{dL}$ or higher. The OR of Q4 with a TG level of $149 \mathrm{mg} / \mathrm{dL}$ or more was higher than that of Q1 to Q3 (OR [95\% Cl]: 1.24 [1.0-1.5]).

\section{Discussion}

The prevalence of CKD in the present study was $17.7 \%$. This value is considered to be close to the prevalence of CKD in general populations in Japan. Another study conducted in 2005 reported that the prevalence of CKD was $13 \%$. This may be due to our subjects being recruited from mass screening examinations and theirs volunteering to participate in the program. [1] [12] The prevalence of CKD in the present study may also have been higher because $63.8 \%$ of our subjects were women, among whom those in their 50s or higher had a high prevalence of CKD.In the present study, significant differences were observed in age, BMI, SBP, DBP, TC, TG, and hyperlipidemia between the CKD and non-CKD groups. Multiple linear regression analysis showed that age, sex, SBP, and hyperlipidemia were associated with reductions in eGFR. Our logistic regression analysis revealed that age, SBP, TG $\geq 150 \mathrm{mg} / \mathrm{dL}$ alone, TC $\geq 220 \mathrm{mg} / \mathrm{dL}$ alone, and high TC and TG were associated with CKD after adjustments for other risk factors. The OR of TC in quartiles for CKD increased linearly, whereas similar changes were not observed for elevations in TG. The risk of developing CKD appeared only at the highest TG quartile ( $\geq 149 \mathrm{mg} / \mathrm{dL})$. Elevations greater than $149 \mathrm{mg} / \mathrm{dL}$, even without increases in TC, may be a risk factor for CKD in this population. These results suggested that hyperlipidemia was a significant risk for CKD, whereas diabetes was not associated with CKD in our subjects recruited between 1992 and 1995. Additionally, the risk of CKD was clearly associated with those whose TG value became $149 \mathrm{mg} / \mathrm{dL}$ or higher, whereas the risk gradually increased in those whose TC value exceeded the normal range.Although the relationship between TC and CKD progression has been extensively examined, few studies have investigated the relationship between TG and CKD according to various lipid profiles and outcomes. [5] [6] Tsuruya et al. reported that the TG/HDL-C ratio was linearly related to CKD. [13] Muntner et al. also indicated that hypertriglyceridemia and low HDL were markers of elevated SCr. [14] Shimizu et al. identified intimamedia thickening and hypertriglyceridemia as risk factors for CKD. [15] A study in Taiwanese adults conducted in 2009 reported a relationship between the TG threshold and CKD, and that the adjusted OR of CKD in subjects with TG $\geq 200 \mathrm{mg} / \mathrm{dL}$ was significantly higher than in those with $\mathrm{TG}<200 \mathrm{mg} / \mathrm{dL}$ (OR [95\%Cl]: 1.901, [1.07-3.36]). [8] Chinese studies reported that TG was a risk factor for CKD in 2020. [16] [17] The TG threshold may differ among countries or regions depending on lifestyles such as eating habits and physical activities. The TG threshold may be used to stratify the risk of developing CKD among different backgrounds.

Hyperlipidemia has been identified as one of the strongest risk factors for CVD. CKD is also an established risk factor for the development of CVD. Therefore, common factors, such as arterial inflammation caused by hyperlipidemia, may contribute to the progression of vascular damage in the 
heart and kidney. [18] On the other hand, lipid abnormalities secondarily caused by glomerulosclerosis have been proposed based on the hypothesis of lipid nephrotoxicity. [19] [20] [21] This lipid cycle is considered to accelerate the progression of CKD, and its mechanism is related to inhibition of the cascade of very-low-density lipoprotein (VLDL) degradation by apoproteins. Altered lipoprotein effects have been reported as one of the main factors affecting nephrotoxicity in hypertriglyceridemia. [22] Recent studies have focused on changes in the n-3 polyunsaturated fatty acid profile and cholesterol metabolism that cannot be evaluated by lipoprotein levels. [23] [24] The management of hyperlipidemia may be important for preventing the progression of CKD and reducing total mortality rates.

Interventional studies on statins recently reported reductions in CVD events and possible renal protection. [4] A meta-analysis by Sandhu et al. revealed that statins suppressed impairments in renal function, increases in urinary proteins, and the onset and recurrence of CVD events. [25] On the other hand, in a meta-analysis by Ting et al., fibrates were shown to reduce CVD events and suppress declines in renal function in subjects with type 2 diabetes and renal impairment. [26]

Limited information is currently available to support the beneficial effects of reductions in TG on the prognosis of CKD. Therefore, further interventional trials are necessary.

Although diabetes is one of the conventional risk factors for CKD, it was not associated with CKD or reductions in eGFR in the present study. As the underlying mechanism, glomerular hyperfiltration has been suggested to increase eGFR in patients with diabetes. [27] [28] Smoking is one of the risk factors for CKD; however, it showed a negative relationship with CKD in the present study. The lower BMI in subjects who smoke may have affected the results obtained. The present study had the following limitations. First, TG data included pre- and post-prandial levels, which introduced a measurement bias. However, the impact of this bias was not large because the subjects in the present study were recruited from a large, multicenter general population. [9] Second, we did not use urinalysis for the definition of the CKD group, because the subjects with positive proteinuria were small. When we performed the same analysis after we included the subjects with positive proteinuria in the CKD group, there was no significant change in the results.

\section{Conclusions}

TC and TG elevations were both independently associated with CKD in a general Japanese population. The relationship with CKD became stronger as TC increased. A TG level of $149 \mathrm{mg} / \mathrm{dL}$ or higher may be the threshold for a relationship with CKD. Since only a few interventional studies have been conducted on the association between TG and CKD, the relationship between TG levels and the development of CKD warrants further research. Interventional studies are expected because the management of TG may prevent the development and progression of CKD.

\section{Abbreviations}


CKD: Chronic kidney disease; TG: triglycerides; eGFR: estimated glomerular filtration rate; TC: total cholesterol; OR: odds ratio; MetS: metabolic syndrome; HDL-C: high-density lipoprotein cholesterol; JMS: Jichi Medical School; CVD: cardiovascular disease; SCr: serum creatinine; BMI: body mass index; SBP: systolic blood pressure; DBP: diastolic blood pressure; BS: blood sugar; FBS: fasting blood sugar; MDRDeGFR: modification of diet in renal disease estimated glomerular filtration rate; SD: standard deviation; SE: standard errors; VLDL: very low-density lipoprotein

\section{Declarations}

\section{Acknowledgements}

We thank all the participants and staff who helped us during this study's process.

\section{Authors' Contributions}

Author contributions were as follows: SI was responsible for data collection. YS and YI performed data analyses. $\mathrm{JI}$ and SI planned the analysis and interpretation of data. YS, YI, and MM designed the research, contributed to data interpretation, and prepared the draft of the manuscript. All authors read and approved the final version of the manuscript.

Corresponding author: Correspondence to Yuko AGO SHIRAISHI

\section{Funding}

This study was funded by a Grant-in-Aid for Scientific Research from the Ministry of Education, Culture, Sports, Science and Technology of Japan; grants from the Foundation for Community Development, Tochigi, Japan; a grant-in-aid from the Ministry of Health, Labor and Welfare of Japan; and Health and Labor Sciences Research Grants (Research on Health Services:

H26-Junkankitou [Seisaku]-Ippan-001; H29-Junkankitou-Ippan-003 and 20FA1002).

\section{Availability of data and materials}

The datasets analyzed during the current study are available from the corresponding author on reasonable request.

\section{Ethics declarations}

Ethics approval and consent to participate

We obtained informed consent individually in writing and all subjects agreed to participate in the present study. 
Each community government and the Institutional Review Board of Jichi Medical University approved this study design and methods (Epidemiology 06-11).

\section{Consent for publication}

All authors have read and approved the content, and they agree to submit it for consideration for publication in the journal.

\section{Competing interests}

The authors declare that they have no competing interests.

\section{References}

1. Imai E, Horio M, Watanabe T, Iseki K, Yamagata K, Hara S, et al. Prevalence of chronic kidney disease in the Japanese general population. Clin Exp Nephrol. 2009;13(6):621-30.

2. Yamagata $K$, Yagisawa T, Nakai S, Nakayama M, Imai E, Hattori M, et al. Prevalence and incidence of chronic kidney disease stage G5 in Japan. Clin Exp Nephrol. 2015;19(1):54-64.

3. Medicine JSfD. Dialysis Medicine Statistics Survey Report 2018. https://docs.jsdt.or.jp/overview/; 2018, Accessed 07. July 2020

4. Cases A, Coll E. Dyslipidemia and the progression of renal disease in chronic renal failure patients. Kidney Int Suppl. 2005(99):S87-93.

5. Thomas G, Sehgal AR, Kashyap SR, Srinivas TR, Kirwan JP, Navaneethan SD. Metabolic syndrome and kidney disease: a systematic review and meta-analysis. Clin J Am Soc Nephrol. 2011;6(10):2364-73.

6. Schaeffner ES, Kurth T, Curhan GC, Glynn RJ, Rexrode KM, Baigent C, et al. Cholesterol and the risk of renal dysfunction in apparently healthy men. J Am Soc Nephrol. 2003;14(8):2084-91.

7. Hou X, Wang C, Zhang X, Zhao X, Wang Y, Li C, et al. Triglyceride levels are closely associated with mild declines in estimated glomerular filtration rates in middle-aged and elderly Chinese with normal serum lipid levels. PLoS One. 2014;9(9):e106778.

8. Lee PH, Chang HY, Tung CW, Hsu YC, Lei CC, Chang HH, et al. Hypertriglyceridemia: an independent risk factor of chronic kidney disease in Taiwanese adults. Am J Med Sci. 2009;338(3):185-9.

9. Ishikawa S, Gotoh T, Nago N, Kayaba K, Jichi Medical School Cohort Study G. The Jichi Medical School (JMS) Cohort Study: design, baseline data and standardized mortality ratios. J Epidemiol. 2002;12(6):408-17.

10. Matsuo S, Imai E, Horio M, Yasuda Y, Tomita K, Nitta K, et al. Revised equations for estimated GFR from serum creatinine in Japan. Am J Kidney Dis. 2009;53(6):982-92.

11. Inker LA, Astor BC, Fox CH, Isakova T, Lash JP, Peralta CA, et al. KDOQI US commentary on the 2012 KDIGO clinical practice guideline for the evaluation and management of CKD. Am J Kidney Dis. 2014;63(5):713-35. 
12. Imai E, Horio M, Iseki K, Yamagata K, Watanabe T, Hara S, et al. Prevalence of chronic kidney disease (CKD) in the Japanese general population predicted by the MDRD equation modified by a Japanese coefficient. Clin Exp Nephrol. 2007;11(2):156-63.

13. Tsuruya K, Yoshida H, Nagata M, Kitazono T, Hirakata H, Iseki K, et al. Association of the triglycerides to high-density lipoprotein cholesterol ratio with the risk of chronic kidney disease: analysis in a large Japanese population. Atherosclerosis. 2014;233(1):260-7.

14. Muntner P, Coresh J, Smith JC, Eckfeldt J, Klag MJ. Plasma lipids and risk of developing renal dysfunction: the atherosclerosis risk in communities study. Kidney Int. 2000;58(1):293-301.

15. Shimizu M, Furusyo N, Mitsumoto F, Takayama K, Ura K, Hiramine S, et al. Subclinical carotid atherosclerosis and triglycerides predict the incidence of chronic kidney disease in the Japanese general population: results from the Kyushu and Okinawa Population Study (KOPS). Atherosclerosis. 2015;238(2):207-12.

16. Zhang YB, Sheng LT, Wei W, Guo H, Yang H, Min X, et al. Association of blood lipid profile with incident chronic kidney disease: A Mendelian randomization study. Atherosclerosis. 2020;300:19-25.

17. Wang X, Chen H, Shao X, Xiong C, Hong G, Chen J, et al. Association of Lipid Parameters with the Risk of Chronic Kidney Disease: A Longitudinal Study Based on Populations in Southern China. Diabetes Metab Syndr Obes. 2020;13:663-70.

18. Mathur S, Devaraj S, Jialal I. Accelerated atherosclerosis, dyslipidemia, and oxidative stress in endstage renal disease. Curr Opin Nephrol Hypertens. 2002;11(2):141-7.

19. Moorhead JF, Chan MK, El-Nahas M, Varghese Z. Lipid nephrotoxicity in chronic progressive glomerular and tubulo-interstitial disease. Lancet. 1982;2(8311):1309-11.

20. Heine GH, Eller K, Stadler JT, Rogacev KS, Marsche G. Lipid-modifying therapy in chronic kidney disease: Pathophysiological and clinical considerations. Pharmacol Ther. 2020;207:107459.

21. Ruan XZ, Varghese Z, Moorhead JF. An update on the lipid nephrotoxicity hypothesis. Nat Rev Nephrol. 2009;5(12):713-21.

22. Vaziri ND, Moradi H. Mechanisms of dyslipidemia of chronic renal failure. Hemodial Int. 2006;10(1):1-7.

23. Shoji T, Kakiya R, Hayashi T, Tsujimoto Y, Sonoda M, Shima H, et al. Serum n-3 and n-6 polyunsaturated fatty acid profile as an independent predictor of cardiovascular events in hemodialysis patients. Am J Kidney Dis. 2013;62(3):568-76.

24. Sonoda M, Shoji T, Kimoto E, Okute Y, Shima H, Naganuma T, et al. Kidney function, cholesterol absorption and remnant lipoprotein accumulation in patients with diabetes mellitus. J Atheroscler Thromb. 2014;21(4):346-54.

25. Sandhu S, Wiebe N, Fried LF, Tonelli M. Statins for improving renal outcomes: a meta-analysis. J Am Soc Nephrol. 2006;17(7):2006-16.

26. Ting RD, Keech AC, Drury PL, Donoghoe MW, Hedley J, Jenkins AJ, et al. Benefits and safety of longterm fenofibrate therapy in people with type 2 diabetes and renal impairment: the FIELD Study. Diabetes Care. 2012;35(2):218-25. 
27. Alicic RZ, Rooney MT, Tuttle KR. Diabetic Kidney Disease: Challenges, Progress, and Possibilities. Clin J Am Soc Nephrol. 2017;12(12):2032-45.

28. Fu WJ, Li BL, Wang SB, Chen ML, Deng RT, Ye CQ, et al. Changes of the tubular markers in type 2 diabetes mellitus with glomerular hyperfiltration. Diabetes Res Clin Pract. 2012;95(1):105-9.

\section{Tables}

Tables 1-3 are available in the Supplementary Files

\section{Figures}

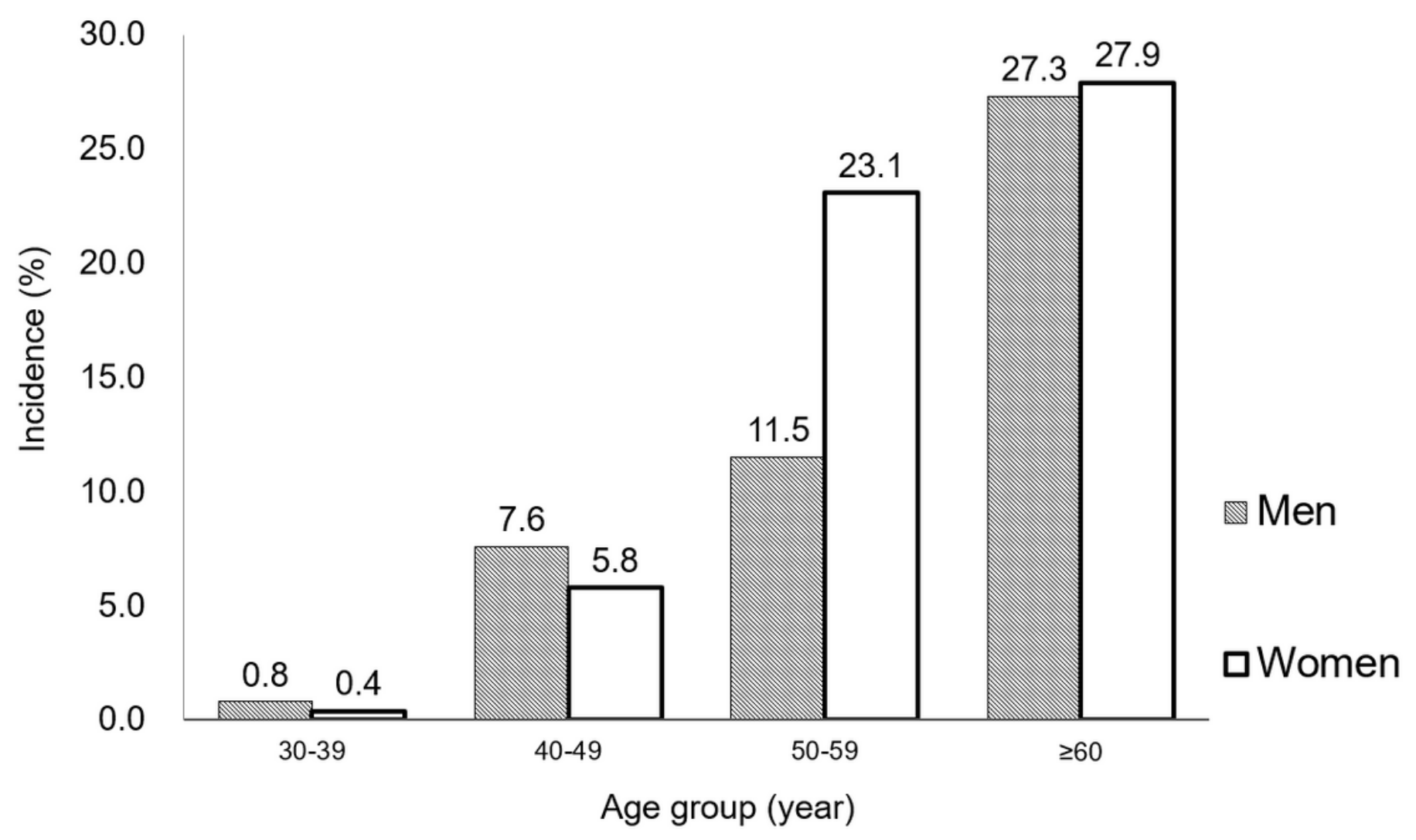

Figure 1

Prevalence of chronic kidney disease by age and sex. The prevalence of CKD by age and sex was shown as percentages in each age group of 30-39, 40-49, 50-59, and $\geq 60$ years. CKD, chronic kidney disease. 


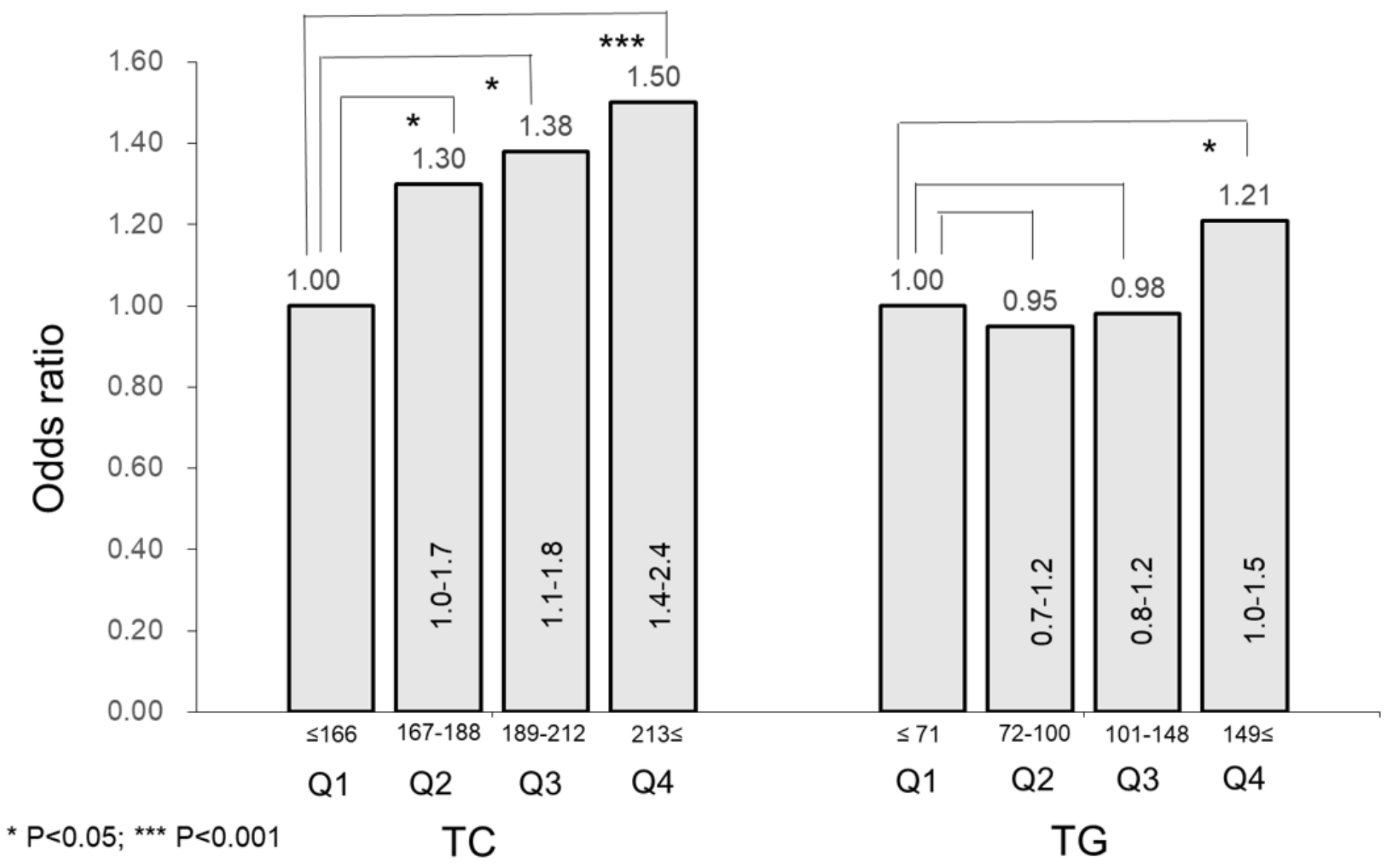

Figure 2

Odds ratios of quartiles of total cholesterol and triglycerides for chronic kidney disease. The odds ratio of quartiles of TC and TG for CKD and 95\% confidence intervals were calculated with adjustments for age, sex, body mass index, systolic blood pressure, diabetes, and current smoking and drinking habits by a logistic regression analysis. TC, total cholesterol; TG, triglycerides; CKD, chronic kidney disease.

\section{Supplementary Files}

This is a list of supplementary files associated with this preprint. Click to download.

- 30383733Tables.xIsx 